\title{
Drug Company
}

National Cancer Institute

\section{Source}

National Cancer Institute. Drug Company. NCI Thesaurus. Code C54149.

A company licensed to discover, develop, market and distribute drugs. 